\title{
¿Influyen las características personales del profesorado en formación en sus actitudes hacia una educación ambiental transformadora?
}

\author{
Do the Personal Attributes of Teachers in Training \\ Affect Their Attitudes Towards Transformative \\ Environmental Education? \\ María-Asunción Lorenzo-Rial' ${ }^{1}$, Uxío Pérez-Rodríguez¹, \\ Mercedes Varela-Losada', Pedro Vega-Marcote ${ }^{2}$ \\ 1 Universidad de Vigo \\ ${ }^{2}$ Universidad de A Coruña
}

\begin{abstract}
Resumen
El desafío ambiental es uno de los principales retos que debe afrontar el profesorado. La finalidad de este estudio fue conocer cómo las características y circunstancias personales pueden influir en las actitudes del futuro profesorado hacia una educación ambiental transformadora. Para ello se empleó una muestra de 889 estudiantes de los Grados en Educación Infantil y Primaria espańoles, analizándose sus puntuaciones en los factores de la escala validada Attitudes Scale toward Environmental Education (ASEE), mediante técnicas de estadística descriptiva e inferencial. Los resultados obtenidos indican que factores como el sexo, el tipo de escuela preuniversitaria y la educación de las familias deben ser tenidos en cuenta, ya que tienen implicaciones prácticas para la formación de los futuros docentes en lo que denominamos una educación ambiental transformadora.
\end{abstract}

Palabras clave: actitudes, características personales, educación ambiental transformadora, escala ASEE, formación del profesorado.

Correspondencia a:

Pedro Vega-Marcote

Facultad de Ciencias de la Educación. Universidad de A Coruña

Campus Elviña. 15701 A Coruña, España

pedro.vega.marcote@udc.es

Financiamiento asociado: Los autores agradecen a los proyectos financiados por el Ministerio de Ciencia, Innovación y Universidades de España (EDU2017-82915-R) y FEDER/Ministerio de Ciencia, Innovación y Universidades de España - Agencia Estatal de Investigación / Proyecto ESPIGA ("Promoviendo el Desarrollo del Pensamiento Crítico y de las dimensiones de Implicación Cognitiva y Emocional de los desempeños Epistémicos en las Clases de Ciencias en la Era de la Posverdad"), referencia PGC2018-096581-B-C22.

(C) 2020 PEL, http://www.pensamientoeducativo.org - http://www.pel.cl 


\begin{abstract}
The environmental problem is one of the main challenges facing teachers. The aim of this study is to find out how personal characteristics and circumstances can influence the attitudes of future teachers to transformative environmental education. For this purpose, a sample of 889 students was used from degree courses in Early Childhood Education and Elementary Education in Spain, analyzing their scores on the factors of the validated questionnaire Attitudes Scale toward Environmental Education (ASEE) by means of descriptive and inferential statistical techniques. The results obtained indicate that factors such as gender, type of pre-university school, and family education should be taken into account, as they have practical implications for training future teachers in what we call Transformative Environmental Education.
\end{abstract}

Keywords: ASEE questionnaire, attitudes, personal attributes, teacher training, transformative environmental education.

\title{
Introducción
}

Los escenarios actuales alertan que estamos ante una emergencia planetaria o crisis sistémica planetaria sin precedentes, de la que la educación no puede ni debe permanecer ajena (Bybee, 1991; Vilches y Gil, 2009; 2015). De hecho, la búsqueda de la sostenibilidad es uno de los principales retos que debe afrontar la educación del siglo XXI (European Commission, EC, 2019).

Este contexto muestra claras evidencias de cómo los seres humanos nos estamos convirtiendo en los protagonistas de las alteraciones que se están produciendo en los procesos que regulan el sistema Tierra, y que recogen términos como cambio ambiental global o antropoceno (Steffen, Broadgate, Deutsch, Gaffney \& Ludwig, 2015), presentes en la literatura científico-experimental de los últimos años. A esto se suma que en la última década numerosos estudios señalan que ya se han superado muchos límites ambientales (Rockström et al., 2009; GEO-6, 2019), lo que nos sitúa ante una situación de incertidumbre, al desconocer las posibles consecuencias de problemas complejos e interrelacionados (Steffen et al., 2015). De ahí que para Melero y Solís (2012) no estemos solo ante una crisis planetaria, sino también de civilizaciones, de modelos de desarrollo económico, social, científico y tecnológico y, fundamentalmente, de educación y de valores.

Detrás de esto se encuentra el modelo socioeconómico dominante, que tal y como señala Stiglitz (2015), es injusto e insostenible, ya que lleva consigo un reparto desigual de los recursos donde la pobreza y las desigualdades se agravan, y una degradación ambiental sin precedentes (Melero y Solís, 2012; Worldwatch Institute, 2015). Al respecto, Gisbert (2007) y Rockström \& Klum (2015) indican que en un planeta con recursos limitados no es posible un crecimiento continuo.

Es por ello que la ONU aprobó en 2015 la Agenda 2030 para el Desarrollo Sostenible. En ella se recoge, a través de sus 17 objetivos y 169 metas, la necesidad de un plan de acción conjunto para poner en marcha programas e iniciativas que ayuden a la creación de sociedades más justas y sostenibles. 


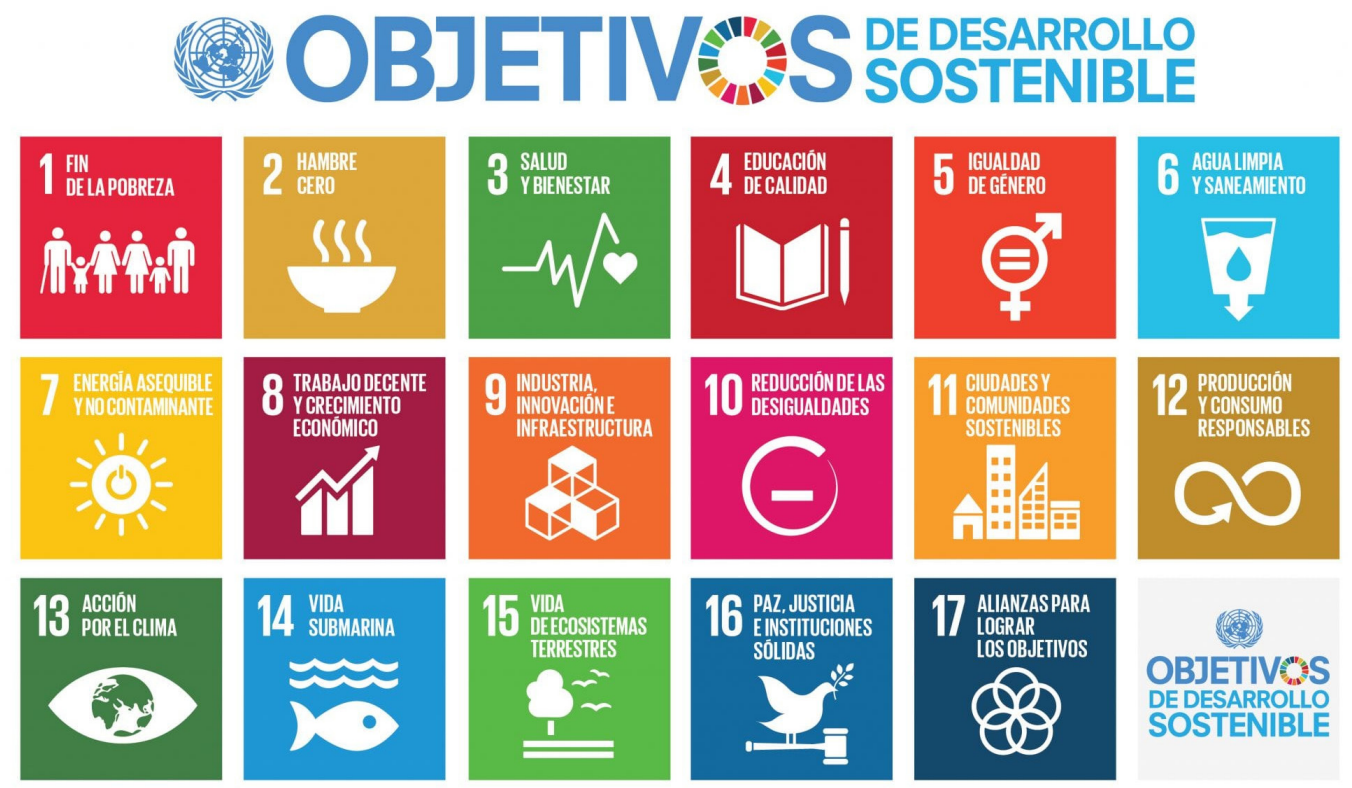

Figura 1. Agenda 2030 para el Desarrollo Sostenible y sus 17 Objetivos (ONU, 2015).

El objetivo 4 de esta agenda indica de manera específica el papel principal de la educación como medio para garantizar una formación inclusiva, equitativa y de calidad que promueva oportunidades de aprendizaje a lo largo de la vida para todas las personas. Respecto de esta formación, Irina Bokova — desde 2009 directora general de la Organización de las Naciones Unidas para la Educación, Unesco, organismo encargado de seguir el progreso en la consecución del objetivo 4-, señala que:

Debemos cambiar fundamentalmente la manera en que concebimos la educación y su función en el bienestar humano y el desarrollo mundial. Ahora más que nunca, la educación tiene la responsabilidad de fomentar el tipo adecuado de competencias, actitudes y comportamientos que llevarán al crecimiento sostenible e inclusivo (Unesco, 2016, página inicial).

En este marco y con los seres humanos como centro, tanto la educación como la educación ambiental de carácter transformador se presentan como un medio necesario para formar personas conscientes y críticas, capaces de valorar los riesgos de las tendencias actuales y de trabajar por un futuro sostenible, en consonancia con la evolución que experimentó en las últimas décadas esta materia (Murga-Menoyo y Novo, 2017).

No obstante, para que esto suceda el modelo educativo actual debe ser revisado ya que, como señalan Álvarez y Vega (2009), promover un desarrollo sostenible desde la educación ambiental implica "redefinir los nuevos escenarios educativos, sus tiempos y ritmos, el papel del profesorado y de todos los actores que intervienen en la práctica escolar, el currículo, su gestión y el ecosistema pedagógico actual” (p. 246).

Esto supone un gran reto para el personal docente y, como señalan Gough (2016) y Wiek, Xiong, Brundiers y Leeiw Van Deer (2014), también para la formación del profesorado, ya que en este proceso reside una de las claves para promover el cambio en las futuras generaciones, al llevar a la práctica propuestas educativas orientadas hacia la sostenibilidad en los centros, e implicando a toda la comunidad educativa. 


\section{El reto de una educación ambiental transformadora orientada hacia la sostenibilidad}

Hoy en día sabemos que la educación en general y las escuelas en particular juegan un papel relevante en la adquisición de conocimientos, habilidades, destrezas y actitudes orientadas hacia la promoción de nuevas formas de vida conscientes y respetuosas con el medio y las personas (Worlwatch Institute, 2015). Pese a ello, el cambio debe venir a partir de una educación ambiental transformadora (en adelante EAT) concebida desde una perspectiva crítica y reflexiva que ponga en cuestionamiento el modelo socioeconómico dominante, para lo cual las escuelas deben doblar sus esfuerzos para que la ciudadanía esté formada e informada. Tal y como indican Milanés, Menezes y Quellis (2019) los establecimientos educativos constituyen un espacio idóneo para favorecer la generación de nuevas ideas, lo que contribuye a la construcción de sociedades más justas y sostenibles al permitir que tanto el profesorado, como el alumnado y la comunidad puedan participar y tomar decisiones respecto de problemas socioambientales de su entorno próximo.

Así, la educación para el cambio debe comenzar en la primera infancia, porque se trata de una época en la que se establecen importantes fundamentos y un deseo de aprender para toda la vida, por lo que se puede provocar que el alumnado se vea como personas capaces de hacer valiosas contribuciones a su comunidad (Mackey, 2012). De este modo, el conocimiento de los problemas socioambientales y la búsqueda reflexiva y participativa de soluciones desde las etapas iniciales es una forma de preparar a los estudiantes para la complejidad de estos problemas que enfrentarán a medida que crecen. Esta implementación gradual ayudaría, igualmente, a hacer frente al gran reto que supone educar para la sostenibilidad ante problemas globales que están interconectados (Kopnina \& Meijer, 2014; Novo, 2006).

Afrontar este reto, como defiende Murga-Menoyo (2015), precisa de un nuevo marco didáctico que promueva el desarrollo de competencias y capacidades necesarias para la creación de una ciudadanía crítica y comprometida con un modelo de desarrollo distinto, al mismo tiempo que se enseña a hacer frente a los desafíos ambientales globales (Leicht, Heiss \& Byun, 2018; Scott, 2009; Unesco, 2017). En este sentido, autores como Olsson, Gericke, Sass y Boeve-de Pauw (2020) defienden que esto solo será posible a través del desarrollo de una competencia para la acción, basada en la comprensión del carácter complejo de los problemas socioambientales, el pensamiento crítico, la responsabilidad individual y social, y la toma de decisiones participativa, de modo que ello ponga en valor el desarrollo de valores proambientales desde el ámbito educativo (Jensen \& Schnack, 2006; Mogensen \& Schnack, 2010; Unesco, 2017).

De este modo, contribuir al desarrollo de esta competencia desde la EAT conlleva, tal y como señalan Jesen y Schnack (2006), comprender las posibles soluciones a los problemas socioambientales actuales, lo que implica promover acciones educativas basadas en el pensamiento complejo (Bonil, Junyent y Pujol, 2010; García, 2004; Morin \& Pakman, 1994; Osorio, 2002), a partir de la necesidad de hacer conscientes a las nuevas generaciones de la importancia de llevar a cabo cambios sociales y estructurales. Esto significa superar la fragmentación del conocimiento y el determinismo de la ciencia, ya que estos impregnan las prácticas pedagógicas y los programas de formación inicial docente (Saheb \& Rodríguez, 2017). De esta manera, la teoría de la complejidad (Morin, 2001) pretende reafirmar la necesidad de reconsiderar la educación, en general y la educación ambiental en particular, al postular una reforma del pensamiento como medio para contextualizar el aprendizaje.

Por otro lado, Tilbury (1995) y Breiting (1997) señalan además que una educación ambiental que fomenta la acción debe promover actitudes críticas y reflexivas, asumiendo que el pensamiento crítico es un eje fundamental para que se produzca la acción, al representar la capacidad que debemos desarrollar para tomar decisiones responsables, pensar con autonomía y formar parte activa de las decisiones culturales, científicas y socioambientales actuales (Solbes y Torres, 2012; Torres y Solbes, 2016). 
De este modo, solo desde una perspectiva holística y participativa se podrá caminar hacia un desarrollo sostenible real y efectivo (Wals, 2014). Al respecto, Vilches y Gil (2015) señalan tres características de este nuevo enfoque de enseñanza, el cual debe ser: interdisciplinar, ya que tiene que abordar retos complejos; transdisciplinar, pues necesita de la participación de toda la ciudadanía; y tener una perspectiva amplia, es decir, ser global y tener un enfoque a corto y largo plazo.

Todo esto implica la movilización de aprendizajes relacionados con aprender a ser, conocer, convivir, hacer y sentir, necesarios para transformar la sociedad (Lorenzo Rial, Álvarez Lires, Arias Correa y Pérez Rodríguez, 2019). Estas competencias deben caracterizarse por fomentar actitudes de responsabilidad, compromiso y acción, por lo que será fundamental la implicación de toda la comunidad educativa (Bonil, Calafell, Granados-Sánchez, Junyent y Tarín, 2012; Jara Campos, 2020), así como la implementación de experiencias didácticas que favorezcan la participación y la toma de decisiones por parte del alumnado, protagonista de los cambios necesarios (Ayerbe López, y Perales Palacios, 2020; Mackey, 2012; Öhman \& Öhman, 2013).

Al respecto, Orr (1993) señala que el primer paso para la construcción de conocimiento es la iniciación a través de un proceso de alfabetización ambiental (environmental literacy) como una forma de abordar el mejoramiento de las cuestiones socioambientales (Tuncer Teksoz, Boone, Tuzun \& Oztekin, 2013), con un enfoque sistémico y transformador, integrando y promoviendo la búsqueda de un modelo de desarrollo socioeconómico, que permita la comprensión de las consecuencias del crecimiento continuo para el planeta (Pérez-Rodríguez, Varela-Losada, Lorenzo-Rial y Vega-Marcote, 2017). Estos esfuerzos debieran lograr que el alumnado adquiera una visión de nuestra ecodependencia, comprendiendo el gran impacto de las actividades antropogénicas en el medio ambiente y en las personas, para que puedan tener conciencia de los principios ecológicos (Braun, Cottrell \& Dierkes, 2018; Ernst \& Theimer, 2011; Evans et al., 2007; Gifford \& Sussman, 2012). En este contexto, Vilches y Gil (2015) hablan de "ciencia de la sostenibilidad" para hacer referencia a las relaciones entre sociedad y sistemas naturales y a la necesidad de reorientar las interacciones entre estos dos ámbitos.

Por último, la escuela y la formación inicial del profesorado deben establecer sinergias entre los medios natural, social y cultural, con la finalidad de integrar el debate ecológico y las implicaciones de las políticas en torno al medio ambiente como cuestiones esenciales para entender el impacto de nuestras acciones (Brody, 1996). La integración de la alfabetización científica ambiental en la educación exige repensar las finalidades de la EAT, revisar los enfoques de los materiales didácticos que perpetúan el modelo actual de desarrollo y reformular críticamente las prácticas docentes, a fin de conocer qué se enseña, cómo se está enseñando y qué se pretende conseguir (Jones, Ramanau, Cross \& Healing, 2010).

\section{La educación ambiental transformadora orientada a la sostenibilidad en la formación}

\section{inicial del profesorado}

No cabe ninguna duda acerca de que el desafío ambiental es uno de los principales retos que debe afrontar la ciudadanía del siglo XXI, y, por consiguiente, también el profesorado (Unesco, 2017).

En este contexto las universidades juegan un papel relevante en la formación de profesionales capaces de adoptar comportamientos y prácticas proambientales que promuevan la conservación y protección del medio ambiente y de las personas (Heyl, Moyano Díaz \& Cifuentes, 2013). No obstante la realidad es que, aunque los currículos escolares y los propios planes de estudio de las universidades hayan incluido la sostenibilidad como eje transversal (Leal Filho et al., 2019), muchos de estos se limitan a la introducción de cambios de corto alcance, al ignorar la necesidad de formular propuestas que tengan en cuenta la urgencia de tomar medidas o la necesidad de poner en cuestión los modelos socioeconómicos actuales, insostenibles y equitativamente injustos (González Gaudiano, 2012). 
En este marco es necesaria una formación docente que promueva un perfil específico de profesorado reflexivo e innovador, capaz de movilizar los conocimientos de su alumnado para ayudarle a asumir su responsabilidad como agentes de cambio (Ull, Piñero, Agut, y Aznar, 2014). Para ello, el docente debe ser capaz de comprender la necesidad de cambiar la manera de enseńar y de aprender, como medio para poder contribuir al desarrollo de las competencias para la sostenibilidad (Wiek, Withycombe \& Redman, 2011).

Este cambio supone superar el modelo de enseñanza tradicional, caracterizada por la transmisión de conocimientos, para centrarnos en un modelo socioconstructivista, que permita formar personas comprometidas, responsables y capaces de actuar y resolver problemas socioambientales (Albareda-Tiana, Vidal-Raméntol, Pujol-Valls \& Fernández-Morilla, 2018). Sin embargo, dentro de la educación ambiental muchas de las prácticas docentes se enmarcan en un enfoque activista, que a pesar de sus bondades aún está caracterizado por la utilización de metodologías tradicionales (Rodríguez y García, 2009). Así, desde un enfoque crítico, Rodríguez (2011) recoge en su tesis doctoral que pese a las buenas intenciones del profesorado que incluye la educación ambiental en sus programaciones, el enfoque didáctico de estas

no propicia un análisis global y sistémico de los problemas ambientales, de la reflexión sobre el papel de las personas y los diversos grupos sociales respecto a los mismos y, por tanto, de la necesidad de adoptar conductas proambientales de forma autónoma y crítica (Rodríguez, 2011, p. 42).

Es por ello que Rodríguez y García (2009), defienden que es necesario buscar modelos constructivistas, que incluyan una reflexión acerca de la finalidad de las actividades propuestas, la formulación y organización de sus contenidos, un diseño de secuencia globalizado y la participación del alumnado al que se dirige la propuesta educativa, para lo cual se debe potenciar en los currículos la capacitación docente en estas temáticas (ÁlvarezGarcía, O., Sureda-Negre \& Comas-Forgas, 2015).

En esta misma línea, muchos estudios llevados a cabo con profesorado en formación inicial (Álvarez-García, Sureda-Negre y Comas-Forgas, 2018; García-Esteban y Murga-Menoyo, 2015; Van Petegem, Blieck, Imbrecht \& Van Hout, 2005) coinciden al señalar las razones por las que este colectivo tiene problemas para implementar una educación ambiental de carácter transformador en sus propuestas para el aula:

- falta de formación específica en la temática,

- escasa experiencia con el uso de métodos didácticos innovadores,

- falta de referencia a elementos curriculares relacionados con la educación ambiental y

- falta de tiempo, lo que implica sobrecarga de trabajo.

Para superar dichas limitaciones, las universidades y centros de formación docente deben dotar al futuro profesorado de herramientas, enfoques y medios necesarios para la implementación de futuras propuestas educativas para el aula, enfocadas en la EAT y que contemplen la interrelación de los problemas socioambientales y la complejidad de diseñar propuestas de carácter global e interdisciplinar. También se deben integrar conocimientos acerca de los problemas socioambientales y el desarrollo sostenible, así como nuevos enfoques para la enseñanza y el aprendizaje, lo que además requiere de una revisión de los planes de estudios actuales (Cebrián y Junyent, 2014; Flores, Ruíz y del Socorro Rayas, 2017). Al respecto, Tilbury (2011) señala que existen tres grandes premisas en el futuro de la enseñanza y el aprendizaje de modelos de desarrollo sostenible: contribuir a promover competencias tecnocientíficas; reformular las relaciones entre seres humanos y naturaleza; e introducir la idea de cambio social. Además, añade que para alcanzar la transformación del sistema es necesario que todas las personas que forman la comunidad educativa reflexionen críticamente acerca del futuro, teniendo en cuenta la complejidad de los cambios, la incertidumbre de sus consecuencias y el reto de que esta nueva situación requiere de un cambio en nuestros valores. 
Todo ello debe formar un profesorado capaz de reformular críticamente sus prácticas docentes (Jones et al., 2010) a fin de introducir metodologías innovadoras que promuevan una EAT, así como repensar su actitud y responsabilidad ante los problemas socioambientales y conocer los fundamentos del desarrollo sostenible, prestando especial atención al papel relevante que tiene el modelo socioeconómico actual en las tendencias socioambientales.

\section{Actitudes del profesorado ante la EAT}

En este contexto, el estudio de las actitudes constituye un elemento esencial de la investigación, ya que estas suelen relacionarse con el comportamiento de las personas (Gifford, 2014). En efecto, en una reciente revisión Marcinkowski y Reid (2019) definen las actitudes como las disposiciones y juicios evaluativos de una persona acerca de un "objeto" (por ejemplo, un ser, una cosa, un acontecimiento, una idea, una cuestión o una acción), que están formadas por la interacción entre componentes cognitivos (conocimientos, creencias, etc.), afectivos (sentimientos, emociones, etc.) y conativos (intención de comportamiento) y que se basan, al menos en parte, en experiencias y situaciones que viven las personas. Así, normalmente se producen como resultado de su interacción con la realidad (Donahue \& Miller, 2006) y derivan de los propios procesos de socialización con otras personas (Kerin, Hartley \& Rudelius, 2009), afectando sus creencias y valores (Bohner \& Wanke, 2002).

Desde esta perspectiva, las actitudes pueden variar con la influencia de factores como la educación, los medios de comunicación y las familias, a lo que se suman otros elementos, como la actualidad y las políticas neoliberales capitalistas (Bentley, Petcovic \& Cassidy, 2019; Schindel Dimick, 2015).

Así, diversos estudios señalan la existencia de una correlación entre las características personales y las actitudes proambientales, que pueden derivar del contexto en cuanto a características demográficas y a factores personales y sociales (Gifford \& Nilsson, 2014; Pavalache-Ilie \& Cazan, 2018). Estos factores también son variables importantes durante la edad escolar. En efecto, desde la psicología cognitiva, social y conductual (Gifford \& Sussman, 2012; Winter \& Koger, 2008; Lehnert, Fiedor, Frajer, Hercik \& Jurek, 2019) suele examinarse la influencia de características personales tales como la edad, el género, la personalidad, el nivel socioeconómico, la residencia urbano-rural, la nación, la religión, la política, los valores, la experiencia, la educación y el conocimiento ambiental. En este sentido, para este estudio hemos tomado como punto de partida la hipótesis de que el profesorado en formación podría presentar actitudes significativamente diferentes de acuerdo con sus características y circunstancias personales, tal y como señala estos autores.

Por otra parte, la literatura recoge un número importante de estudios que muestran cómo la mayoría del profesorado presenta una alta conciencia ambiental (Boubonari, Markos \& Kevrekidis, 2013; Forbes \& Zint, 2010; Olsson, Gericke, Boeve-de Pauw, Berglund \& Chang, 2019; Ull, Martínez-Agut, Piñeiro \& Aznar-Minguet, 2014; Vega-Marcote \& Álvarez, 2012; Yavetz, Goldman \& Pe'er, 2009), pero esto no parece ser suficiente para conseguir una capacitación adecuada para enseñar una EAT. En este sentido, como destaca Aarnio-Linnanvuori (2019) es necesario seguir investigando cuáles son los factores que influyen en la toma de decisiones educativas del profesorado, con el fin de diseńar modelos de formación innovadores que mejoren su práctica docente y favorezcan una ciudadanía consciente y responsable. Es por todo ello que conocer las actitudes ambientales que caracterizan al futuro profesorado es fundamental para identificar su rol en la enseñanza de la educación ambiental, su actitud frente a los problemas, y su importante papel en la mitigación de estos.

De este modo, la finalidad de este estudio fue conocer cómo las características y circunstancias personales pueden influir en las actitudes del futuro profesorado hacia una EAT. Para ello recurrimos a una muestra de estudiantes de los Grados en Educación Infantil y Primaria españoles, analizando sus puntuaciones en los factores de la escala validada Attitudes Scale toward Environmental Education (ASEE) mediante técnicas de estadística descriptiva e inferencial. 


\section{Método}

\section{Metodología}

El presente estudio fue de tipo cuantitativo y su enfoque se asentó sobre las bases del paradigma pospositivista, admitiendo que los sistemas de valores, la cultura y otras circunstancias influencian nuestra percepción del mundo de diferentes maneras (Phillips \& Burbules, 2000). Desde este punto de vista, se intentó realizar una aproximación a las cuestiones de interés de la forma más objetiva y sistemática posible, aunque desde la consciencia de las limitaciones de este planteamiento (Treagust, Won, \& Duit, 2014). En concreto, lo que se pretendió con la investigación fue cuantificar y medir patrones que permitieran la formulación de tendencias susceptibles de ser extrapoladas a otros contextos o poblaciones. En este marco, se utilizó el análisis estadístico como herramienta de medición.

Por otro lado, se tomaron como referencia la Declaración sobre la ciencia y el uso del saber cientifico (Unesco, 1999) así como la Guia de buenas prácticas en investigación de la Universidad de Vigo (aprobada en el Consejo de Gobierno del 20 de marzo de 2018), atendiendo a las directrices indicadas en investigaciones realizadas con seres humanos. En este caso, el tratamiento de los datos se realizó previo consentimiento informado de las personas participantes y se resguardaron sus datos personales en coherencia con el protocolo ético de investigación informado durante el proceso de recolección de antecedentes; complementariamente, los resultados se presentan de forma genérica, de manera de asegurar el anonimato y confidencialidad de los involucrados.

\section{Participantes}

La muestra se seleccionó de forma no probabilística, siguiendo criterios de disponibilidad y estuvo formada por 889 estudiantes de los grados en Educación Primaria (54,4\%) y Educación Infantil (45,6\%) de dos universidades españolas (Vigo y A Coruña). El 83,4\% de los participantes fueron mujeres y el 16,6\% hombres.

\section{Instrumento}

Como instrumento para la recogida de datos se empleó la Escala de Actitudes hacia la Educación Ambiental (Attitudes Scale toward Environmental Education, ASEE) (ver Apéndice), diseñada y validada por Pérez-Rodríguez et al. (2017), que corresponde a una escala de tipo Likert de 18 ítems con cinco niveles de respuesta, los que proporcionaron información acerca de las actitudes hacia:

- la problemática ambiental, indagando en sus actitudes respecto del cambio climático (un problema socioambiental complejo), su responsabilidad ambiental individual, sus maneras de tomar decisiones y el modelo socioeconómico imperante.

- un modelo educativo de carácter transformador basado en el rol del profesorado y en una metodología de tratamiento de la información y resolución de problemas, que busca desarrollar capacidades de participación, reflexión, pensamiento crítico, toma de decisiones e implicación de la comunidad en el alumnado.

El instrumento tuvo una fiabilidad adecuada $(\alpha=, 804$, glb $=, 875$ y $\Omega=, 810)$. La muestra empleada para la validación se dividió en dos, realizándose con una de ellas un análisis de componentes principales y, con la otra, análisis factoriales confirmatorios comparando diversos modelos explicativos. El resultado fue una estructura factorial con cinco factores interrelacionados bien definidos, donde los dos ámbitos descritos se ven bien representados (Ver Tabla 1). El modelo seleccionado tuvo índices de ajuste adecuados por lo que respecta al análisis factorial confirmatorio $\left(\chi^{2} / g l=1,47, \mathrm{AIC}=312,16, \mathrm{CFI}=, 955, \mathrm{RMSEA}=, 033\right)$. 
Tabla 1

Descripción de los factores que forman la escala ASEE

\begin{tabular}{|c|c|c|c|c|}
\hline Factor & Código & $\begin{array}{l}\text { Número } \\
\text { de ítems }\end{array}$ & $\begin{array}{l}\text { Varianza } \\
\text { explicada }\end{array}$ & Descripción de los ítems \\
\hline $\begin{array}{l}\text { Transformative Environmental } \\
\text { Education (EAT) }\end{array}$ & TEE & 5 & $24,7 \%$ & $\begin{array}{l}\text { Incluye ítems relacionados con la necesidad } \\
\text { de tratar la educación ambiental en la } \\
\text { escuela, la implicación de la comunidad y el } \\
\text { desarrollo de habilidades en el aula (como la } \\
\text { participación o la toma de decisiones). }\end{array}$ \\
\hline $\begin{array}{l}\text { Environmental problems } \\
\text { (Problemas ambientales) }\end{array}$ & ENP & 4 & $8,5 \%$ & $\begin{array}{l}\text { Sus ítems hacen referencia a las actitudes } \\
\text { frente a un problema socioambiental } \\
\text { complejo: el cambio climático. }\end{array}$ \\
\hline $\begin{array}{l}\text { Transformative methodology } \\
\text { based on participation and } \\
\text { critical thinking } \\
\text { (Metodología trasformadora } \\
\text { basada en participación y } \\
\text { pensamiento crítico) }\end{array}$ & PCT & 4 & $6,8 \%$ & $\begin{array}{l}\text { Sus ítems se refieren a la metodología y al } \\
\text { rol del profesorado en el marco de una EAT. }\end{array}$ \\
\hline $\begin{array}{l}\text { Individual environmental } \\
\text { responsibility } \\
\text { (Responsabilidad ambiental } \\
\text { individual) }\end{array}$ & IER & 3 & $6,4 \%$ & $\begin{array}{l}\text { Comprende ítems relacionados con } \\
\text { la responsabilidad individual ante los } \\
\text { problemas ambientales y la forma en que se } \\
\text { produce la toma de decisiones. }\end{array}$ \\
\hline $\begin{array}{l}\text { Prevailing socioeconomic } \\
\text { model } \\
\text { (Modelo socioeconómico } \\
\text { imperante) }\end{array}$ & SEM & 2 & $5,9 \%$ & $\begin{array}{l}\text { Sus ítems hacen referencia al modelo } \\
\text { socioeconómico. }\end{array}$ \\
\hline
\end{tabular}

Fuente: Elaboración propia sobre la base de Pérez-Rodríguez et al. (2017).

En el cuestionario también se recabó información acerca de las características y circunstancias personales del alumnado: edad, sexo, titulación, curso, opción de bachillerato cursada, tipo de centro de procedencia y nivel de estudios de la madre y el padre.

\section{Procedimiento de administración}

El instrumento se administró en formato papel. Fue creado con el software de reconocimiento óptico de marcas SDAPS versión 1.1.7 para Linux (Berg, 2014). El alumnado voluntario lo cubrió durante sesiones lectivas presenciales bajo la supervisión de profesorado que proporcionó indicaciones y aclaró las consultas. El reconocimiento de las respuestas se llevó a cabo con el mismo programa.

\section{Variables}

Las variables utilizadas en el estudio se describen en la Tabla 2. Como variables independientes cualitativas dicotómicas se emplearon las circunstancias y características personales del profesorado en formación, mientras que como variables dependientes cuantitativas se contemplaron las puntuaciones en los cinco factores de la ASEE. 
Tabla 2

Variables independientes y dependientes del estudio

Variables independientes

\begin{tabular}{|c|c|}
\hline Variable & Valores posibles \\
\hline \multirow{2}{*}{ Sexo } & Mujer \\
\hline & Hombre \\
\hline \multirow{2}{*}{ Edad } & 22 años o menos \\
\hline & Más de 22 años \\
\hline \multirow{2}{*}{ Titulación } & Grado en Educación Infantil \\
\hline & Grado en Educación Primaria \\
\hline \multirow{2}{*}{ Curso } & Primera mitad de la titulación \\
\hline & Segunda mitad de la titulación \\
\hline \multirow{2}{*}{ Bachillerato cursado } & Científico \\
\hline & No científico \\
\hline \multirow{2}{*}{ Titularidad } & Centro público \\
\hline & Centro privado \\
\hline \multirow{2}{*}{ Religiosidad } & Centro laico \\
\hline & Centro religioso \\
\hline \multirow{2}{*}{ Estudios de la madre } & Sin estudios o estudios primarios \\
\hline & Estudios secundarios o universitarios \\
\hline \multirow{2}{*}{ Estudios del padre } & Sin estudios o estudios primarios \\
\hline & Estudios secundarios o universitarios \\
\hline \multicolumn{2}{|l|}{ Variables dependientes } \\
\hline Variable & Valores posibles \\
\hline $\begin{array}{l}\text { TEE } \\
\text { Transformative Environmental Education (EAT) }\end{array}$ & Valores comprendidos entre 1 y 5 \\
\hline $\begin{array}{l}\text { ENP } \\
\text { Environmental problems } \\
\text { (Problemas ambientales) }\end{array}$ & Valores comprendidos entre 1 y 5 \\
\hline $\begin{array}{l}\text { PCT } \\
\text { Transformative methodology based on participation and critical thinking } \\
\text { (Metodología trasformadora basada en participación y pensamiento crítico) }\end{array}$ & Valores comprendidos entre 1 y 5 \\
\hline $\begin{array}{l}\text { IER } \\
\text { Individual environmental responsibility } \\
\text { (Responsabilidad ambiental individual) }\end{array}$ & Valores comprendidos entre 1 y 5 \\
\hline $\begin{array}{l}\text { SEM } \\
\text { Prevailing socioeconomic model } \\
\text { (Modelo socioeconómico imperante) }\end{array}$ & Valores comprendidos entre 1 y 5 \\
\hline
\end{tabular}

Fuente: Elaboración propia. 


\section{Análisis estadísticos}

Como hemos indicado, las características y circunstancias personales del profesorado en formación que conforma la muestra se codificaron en las variables independientes en forma dicotómica. Las variables dependientes corresponden a las actitudes de interés en este estudio, medidas a través de la escala ASEE. Se presentarán las puntuaciones medias de cada factor para cada nivel de las variables independientes.

Dado que deseábamos conocer cómo las características y circunstancias personales podían influir en las actitudes del futuro profesorado hacia una EAT, quisimos evaluar el grado de asociación entre todos los pares de asociaciones de variables independientes y dependientes. En este caso, el procedimiento estadístico inferencial fue, por tanto, realizar comparaciones entre las puntuaciones que obtuvo cada uno de los grupos de estudiantes en los factores de la escala, analizando si existían diferencias significativas. Con tal fin, se realizaron pruebas de comparación de medias entre dos muestras independientes con el software SPSS versión 20 para Windows (IBM, 2011).

\section{Resultados}

Tras realizar análisis previos con los datos apreciamos que no siempre se cumplían los supuestos necesarios para realizar la prueba paramétrica t de Student, ya que en algunos casos resultaban significativas las pruebas de normalidad (Kolmogorov-Smirnov y Shapiro-Wilk) y homocedasticidad (prueba de Levene). Por tanto, se utilizó el test no paramétrico de Mann-Whitney, que es más robusto y no requería del cumplimiento de dichos supuestos.

En la Tabla 3 se muestran las puntuaciones medias por factor para cada uno de los valores de las variables dicotómicas de características y circunstancias personales, indicándose los casos en los que había diferencias significativas. Se aprecian ahí diferencias significativas en, al menos, una de las puntuaciones medias en los factores para todas las variables independientes, exceptuando el caso referente al tipo de bachillerato cursado. Asimismo, todas las variables dependientes se vieron afectadas por varias variables independientes.

Tabla 3

Puntuaciones medias por factor y variables de caracteristicas y circunstancias personales

\begin{tabular}{llccccc} 
& & TEE & ENP & PCT & IER & SEM \\
\hline \multirow{2}{*}{ Sexo } & Mujer & $4,38^{* *}$ & $4,23^{* *}$ & $4,12^{* *}$ & $3,51^{* *}$ & $3,39^{* *}$ \\
\cline { 2 - 7 } Edad & Hombre & 4,21 & 3,90 & 3,83 & 3,33 & 3,26 \\
\hline \multirow{2}{*}{ Titulación } & 22 años o menos & 4,32 & 4,19 & 4,06 & 3,43 & 3,38 \\
\cline { 2 - 7 } & Más de 22 ańos & $4,44^{* *}$ & 4,15 & 4,12 & $3,63^{* *}$ & 3,36 \\
\hline \multirow{2}{*}{ Curso } & Grado en Ed. Infantil & 4,40 & $4,24^{*}$ & $4,14^{*}$ & 3,52 & $3,46^{* *}$ \\
\cline { 2 - 7 } & Grado en Ed. Primaria & 4,31 & 4,12 & 4,02 & 3,45 & 3,30 \\
\hline \multirow{2}{*}{$\begin{array}{l}\text { Bachillerato } \\
\text { cursado }\end{array}$} & $\begin{array}{l}\text { Primera mitad de la } \\
\text { titulación }\end{array}$ & 4,28 & 4,12 & 3,95 & 3,47 & 3,29 \\
\cline { 2 - 7 } & Segunda mitad de la & $4,46^{* *}$ & $4,26^{* *}$ & $4,27^{* *}$ & 3,50 & $3,51^{* *}$ \\
\cline { 2 - 7 } Titularidad & Citulación & 4,35 & 4,21 & 4,10 & 3,57 & 3,45 \\
\cline { 2 - 6 } & No científico & 4,31 & 4,16 & 4,06 & 3,44 & 3,34 \\
\hline
\end{tabular}




\begin{tabular}{|c|c|c|c|c|c|c|}
\hline \multirow{2}{*}{ Religiosidad } & Centro laico & $4,37^{*}$ & $4,20^{* *}$ & 4,09 & $3,51^{* *}$ & $3,43^{* *}$ \\
\hline & Centro religioso & 4,26 & 4,01 & 3,96 & 3,32 & 3,15 \\
\hline \multirow{2}{*}{$\begin{array}{l}\text { Estudios de la } \\
\text { madre }\end{array}$} & Sin estudios / primarios & 4,38 & $4,24^{*}$ & $4,14^{*}$ & 3,50 & 3,42 \\
\hline & Secundarios / universitarios & 4,33 & 4,13 & 4,03 & 3,47 & 3,34 \\
\hline \multirow{2}{*}{$\begin{array}{l}\text { Estudios del } \\
\text { padre }\end{array}$} & $\begin{array}{l}\text { Sin estudios / } \\
\text { Primarios }\end{array}$ & 4,36 & 4,21 & 4,10 & 3,52 & $3,45^{*}$ \\
\hline & Secundarios / universitarios & 4,34 & 4,15 & 4,05 & 3,45 & 3,32 \\
\hline
\end{tabular}

Nota: Se marcan con asteriscos los casos en los que hay diferencias significativas $\left({ }^{*} \mathrm{p}<, 05,{ }^{* *} \mathrm{p}<, 01\right.$, contraste bilateral) en la prueba de Mann-Whitney. Los asteriscos se agregan en el mayor de los valores correspondientes a cada variable dicotómica.

Fuente: Elaboración propia.

En concreto, la relación entre variables independientes y factores muestra, tal y como se recoge en la Tabla 3, que tanto las características personales (sexo y edad), como el tipo de centro escolar de procedencia (titularidad y/o religiosidad) y los estudios de los progenitores, pueden ser relevantes, a lo que se suman los estudios universitarios que cursan los participantes (titulación y curso).

A modo de síntesis, en relación con las características personales, encontramos que las profesoras en formación muestran una actitud más positiva en todos los factores respecto de sus binomios hombres, mientras que en la variable edad, los datos indican que existe una mejora significativa a medida que pasan los años, aunque solo en lo concerniente a la integración de metodologías innovadoras y a la asunción de responsabilidad ambiental individual.

En cuanto a la influencia que pueda tener el tipo de centro escolar de procedencia del profesorado, observamos diferencias entre las personas que proceden de centros públicos y aquellas que lo hacen de centros privados, teniendo puntuaciones significativamente más altas el estudiantado de centros públicos en todos los factores estudiados. Lo mismo ocurre al comparar los resultados de centros laicos y religiosos: las puntuaciones son significativamente mayores en el caso del alumnado de centros laicos en casi todas las variables de interés.

Los resultados de este estudio también indican que a medida que el profesorado avanza de curso tiene una actitud más positiva, de manera que se observan cambios significativos en su actitud en casi todos los factores.

Asimismo se aprecian diferencias entre estudiantes de los distintos grados universitarios. En este caso, parece que el futuro profesorado de Educación Infantil está más sensibilizado con la necesidad de afrontar el reto del cambio climático, metodológico y del modelo socioeconómico respecto del de Primaria. Así, sus puntuaciones son significativamente más altas en tres de los cinco factores analizados.

Otros aspectos que parecen tener influencia son los estudios de los progenitores. Por ejemplo, se observaron diferencias significativas en cuanto a que el estudiantado cuyas madres contaban con un menor nivel de estudios tenía una actitud más positiva frente a problemas complejos como el cambio climático o la necesidad de cambiar el rol y el enfoque metodológico en el marco de la EAT.

Lo mismo ocurrió con el bachillerato cursado. Al contrario de lo que se podría pensar, no se observaron diferencias significativas entre el estudiantado que cursó un bachillerato tecnocientífico del que lo hizo en una rama de artes, letras o mixto. 
Por último, en este análisis es necesario poner en primer plano aquellos resultados que pueden dar pistas acerca de aspectos que deben ser mejorados en la formación docente. Llaman la atención los resultados de los factores referidos a la "responsabilidad ambiental individual" y al "modelo socioeconómico imperante", que presentan valores en torno al 3\% (bastante inferiores al resto), y que reflejan una postura de desafección.

\section{Discusión}

De estos resultados se aprecia que conocer las actitudes ambientales del profesorado en formación inicial resulta de gran interés, así como lo es también entender la relación que existe entre estas actitudes y sus características y circunstancias personales.

En este sentido, la investigación sugiere que algunas de estas particularidades pueden influir significativamente en la actitud de los futuros docentes hacia una EAT. En efecto, las mujeres muestran aquí una actitud más positiva que los hombres, resultado que señalan muchos otros estudios (Bord y O'Connor, 1997; Goldsmith, Feygina \& Jost, 2013; Hunter, Hatch \& Johnson, 2004) en los que, además, se indica que estas diferencias se basan en la teoría de socialización de género, es decir, en la existencia de discrepancias en la construcción de identidades a partir del sexo (McCright \& Xiao, 2014; Olsson y Gericke, 2017).

Respecto del estudio de la influencia que pueda tener el centro de procedencia preuniversitario, el estudiantado de centros públicos presentó en este estudio puntuaciones significativamente más altas que los alumnos provenientes de centros privados. En este caso parece no existir consenso en la literatura, ya que mientras algunas investigaciones muestran la presencia de una relación entre un nivel socioeconómico alto y una mayor preocupación ambiental (Marquart-Pyatt \& Petrzelka, 2008; Stevenson, Peterson \& Bondell, 2019), otras sin embargo resaltan el hecho de que esto no demuestra un mayor conocimiento ni un mejor comportamiento que los estudiantes de escuelas públicas (Barazarte Castro, Neaman, Vallejo y García Elizalde, 2014). Esta diversidad de resultados puede derivar de varios supuestos, por un lado, referentes a las características del contexto o al tipo de metodología empleada para acercar la educación ambiental a los estudiantes o, por otro, al hecho de que el personal docente de escuelas privadas en cierta medida puede tener condicionada su práctica educativa (Agirreazkuenaga, 2019). El carácter religioso del centro de formación, en tanto, también ha resultado significativo en este estudio: pesquisas previas han mostrado la misma variación en cuanto a la procedencia de centros preuniversitarios laicos (Arbuckle \& Konisky, 2015), al concluir que las creencias religiosas pueden influir de manera negativa en las actitudes ambientales (Alkaher \& Carmi, 2019).

En relación con la formación docente, este estudio sugiere que a medida que se avanza en la carrera de magisterio las actitudes ambientales mejoran. Otras investigaciones, sin embargo, muestran que ello no siempre es así (Álvarez-García et al., 2018; Pe’er, Goldman \& Yavetz, 2007; Yavetz et al., 2009). Cuando hablamos de especialidades en la formación inicial, el futuro profesorado de Infantil de este estudio parece más sensibilizado que el de Primaria. Estudios como el de Yurt, Cevher-Kalburan y Kandır (2010) señalan que son escasas las referencias en la literatura a las actitudes ambientales del profesorado de Educación Infantil, ya que la mayoría se centra principalmente en el profesorado de Educación Primaria y Secundaria. Un estudio español (Ull et al., 2014) resalta la necesidad de reforzar las competencias en sostenibilidad como medio para promover el desarrollo sostenible en los grados de Educación Infantil y Primaria, para lo que señalan sería necesaria una reformulación de los planes de estudio.

Otros aspectos que parecen tener cierta influencia son los estudios de los progenitores, los cuales han sido señalados a veces en la literatura como necesarios de considerar (Gifford \& Nilsson, 2014), aun cuando en esta investigación mostraron un efecto limitado. 
Por otra parte, al contrario de lo que se podría pensar, no se observan diferencias significativas entre el estudiantado que cursó un bachillerato tecnocientífico del que lo hizo en una rama de artes, letras o mixto. De esta forma, el profesorado en formación encuestado parece no tener una actitud más positiva por haber cursado materias relacionadas con la comprensión del medio natural respecto de aquellos que no lo han hecho. Aunque diversos estudios señalan diferencias (Müderrisoğlu \& Altanlar, 2011; Tehrani, Karbassi, Monavari \& Mirbagheri, 2010; Thapa, 1999) en las actitudes y comportamientos ambientales debido a la educación previa, en España los materiales curriculares tienden a reproducir el modelo de desarrollo actual, lo que sumado a la falta de formación específica del profesorado en temáticas medioambientales, lleva a que no se establezcan relaciones entre el modelo de consumo, el de producción y la necesidad de cambios en estos para promover el desarrollo sostenible (Hernández, Burgui, Velázquez y Corrales, 2018). A esto se suma que la educación ambiental no figura explícitamente como tal en los planes de estudio de la educación obligatoria, sino que aparece mencionada en los objetivos generales de los currículos de manera anecdótica.

En cuanto al análisis de los resultados derivados de los factores, se aprecia la existencia de valores más bajos encontrados en ítems relacionados con la responsabilidad individual ante problemas ambientales y con aquellos referidos al modelo de desarrollo socioeconómico. Al parecer esta indiferencia puede estar relacionada con la falta de formación específica y de planes de estudios "ambientalizados", que pongan el foco en la complejidad y rapidez de las interconexiones existentes entre los ecosistemas, las sociedades y las economías y den referencias de estas sinergias (Rieckmann, 2012; Ull et al., 2014). De hecho, los resultados relativos a la responsabilidad ambiental individual muestran que no parece existir un cambio de actitud significativo durante el proceso de formación y tampoco aparece el efecto de la titulación ni de la opción de bachillerato cursada. Así, estos aspectos deberían ser especialmente considerados en el diseño de modelos educativos enmarcados en una EAT.

\section{Implicaciones educativas}

Creemos que la recopilación de información como la de este estudio puede contribuir a desarrollar itinerarios didácticos que permitan la evolución de ideas, creencias y actitudes hacia modelos más comprometidos con el medio ambiente (Yus Ramos, 1993). A tal fin, estos nuevos modelos deben tener en cuenta el interés del futuro profesorado por un cambio metodológico y el papel del profesorado para una EAT, en la que las responsabilidades sociales e individuales marquen el contexto didáctico y sirvan como punto de partida para su diseño.

En los últimos años han surgido numerosas propuestas metodológicas en esta dirección, que conllevan un aprendizaje de la EAT que une teoría y práctica educativa. Algunos ejemplos pueden ser los modelos de Cebrián y Junyent (2015) de resolución de problemas sociales y ambientales, y las ideas de Leal Filho, Shiel y Paco, (2016) de aprendizaje por proyectos. También son interesantes los planteamientos de ecometodología (VegaMarcote \& Álvarez, 2012) y las experiencias de aprendizaje servicio como las de Barth, Adomßent, Fischer, Richter y Rieckmann (2014). Creemos que estas ideas son capaces de promover cambios en la educación del profesorado encaminados a formar maestros y maestras capaces de educar a un alumnado crítico, responsable $\mathrm{y}$ respetuoso con el medio ambiente y las personas.

\section{Conclusiones}

La educación y las escuelas deben hacer frente a los retos que suponen los problemas socioambientales. Desde esta perspectiva, la predisposición del profesorado hacia un enfoque transformador de la educación ambiental parece una seńal de avance: por un lado, para superar al analfabetismo ambiental, caracterizado por la falta de 
conocimientos acerca de la complejidad del funcionamiento del sistema Tierra y la escasa compresión en relación con la interdependencia entre los sistemas naturales y los modelos socioeconómicos y, por otro, replantearse su actitud y compromiso hacia los fundamentos que sustentan el desarrollo sostenible.

En definitiva, este estudio ha permitido explorar cómo las características y circunstancias personales del profesorado pueden influir en sus actitudes ambientales que, tal y como señalan Skamp (2009), son muy importantes para poder conocer la evolución de las ideas y creencias que muestra el estudiantado en los diferentes niveles de educación. Así, los resultados permiten apreciar diferencias en relación con el sexo y el tipo de centro escolar preuniversitario, donde su carácter público/privado y su orientación religiosa son factores a tener cuenta. También son interesantes — sobre todo por sus implicaciones en la formación docente—, los datos obtenidos en relación con la modesta responsabilidad individual y la postura indiferente ante un modelo de desarrollo socioeconómico injusto e insostenible. Aunque también es necesario resaltar que todos estos resultados deben ser ampliados mediante estudios de tipo cualitativo y mixto en futuras investigaciones. Utilizar un enfoque cualitativo nos puede ayudar a conocer el significado que cada persona atribuye a su experiencia y, como resultado de ello, cómo la entiende y cómo la explica (Creswell, 2014), de forma que puede contribuir a estudiar en profundidad los datos obtenidos en el contexto de esta investigación y ayudar a explicar las discordancias con otros estudios.

De esta manera, las evidencias mostradas en esta y otras investigaciones en relación con la relevancia de integrar la EAT en la formación inicial del profesorado deben servir para mejorar la educación de una ciudadanía del siglo XXI que sea capaz de afrontar los problemas presentes y futuros, tanto a nivel local como global.

El artículo original fue recibido el 14 de mayo de 2020

El artículo revisado fue recibido el 24 de julio de 2020

El artículo fue aceptado el 28 de agosto de 2020

\section{Referencias}

Aarnio-Linnanvuori, E. (2019). How do teachers perceive environmental responsibility? Environmental Education Research, 25(1), 46-61. https://doi.org/10.1080/13504622.2018.1506910

Agirreazkuenaga, L. (2019). Embedding sustainable development goals in education. Teachers' perspective about education for sustainability in the Basque Autonomous Community. Sustainability, 11(5), 1-17. https://doi.org/10.3390/su11051496

Albareda-Tiana, S., Vidal-Raméntol, S., Pujol-Valls, M., \& Fernández-Morilla, M. (2018). Holistic approaches to develop sustainability and research competencies in pre-service teacher training. Sustainability, 10(10), 3698-4007. https://doi.org/10.3390/su10103698

Alkaher, I. \& Carmi, N. (2019). Is population growth an environmental problem? Teachers' perceptions and attitudes towards including it in their teaching. Sustainability, 11(7), 1994. https://doi.org/10.3390/su11071994

Álvarez, P. y Vega, P. (2009). Actitudes ambientales y conductas sostenibles. Implicaciones para la educación ambiental. Revista de Psicodidáctica, 14(2), 245-260. Recuperado de https://www.redalyc.org/pdf/175/17512724006.pdf

Álvarez-García, O., Sureda-Negre, J., \& Comas-Forgas, R. (2015). Environmental education in pre-service teacher training: A literature review of existing evidence. Journal of Teacher Education for Sustainability, 17(1), 72-85. https://doi.org/10.1515/jtes-2015-0006

Álvarez-García, O., Sureda-Negre, J., y Comas-Forgas, R. (2018). Evaluación de las competencias ambientales del profesorado de primaria en formación inicial: estudio de caso. Enseñanza de las Ciencias: Revista de Investigación y

Experiencias Didácticas, 36(1), 117-141. https://doi.org/10.5565/rev/ensciencias.2338 
Arbuckle, M. B. \& Konisky, D. M. (2015). The role of religion in environmental attitudes. Social Science Quarterly, 96(5), 1244-1263. https://doi.org/10.1111/ssqu.12213

Ayerbe López, J. y Perales Palacios, F. J. (2020). "Reinventa tu ciudad": aprendizaje basado en proyectos para la mejora de la conciencia ambiental en estudiantes de secundaria. Enseñanza de las Ciencias, 38(2), 181-203. https://doi.org/10.5565/rev/ensciencias. 2812

Barazarte Castro, R., Neaman, A., Vallejo Reyes, F., y García Elizalde, P. (2014). El conocimiento ambiental y el comportamiento proambiental de los estudiantes de enseñanza media, en la Región de Valparaíso (Chile). Revista de Educación, 364, 66-92. Recuperado de https://dialnet.unirioja.es/servlet/articulo?codigo=4684411\&orden=1\&info=link

Barth, M., Adomßent, M., Fischer, D., Richter, S., \& Rieckmann, M. (2014). Learning to change universities from within: A service-learning perspective on promoting sustainable consumption in higher education. Journal of Cleaner production, 62, 72-81. https://doi.org/10.1016/j.jclepro.2013.04.006

Bentley, A. P., Petcovic, H. L., \& Cassidy, D. P. (2019). Development and validation of the anthropogenic climate change dissenter inventory. Environmental Education Research, 25(6), 867-882. https://doi.org/10.1080/13504622.2016.1250150

Berg, B. (2014). SDAPS: Scripts for Data Acquisition with Paper-based Surveys (Version 1.1.7) [Computer software]. https://sdaps.org/

Bohner, G. \& Wanke, M. (2002). Attitudes and attitude change. East Sussex: Psychology Press.

Bonil, J., Calafell, G., Granados Sánchez, J., Junyent, M., y Tarín, R. M. (2012). Un modelo formativo para avanzar en la ambientalización curricular. Profesorado, 16(2), 145-163. Recuperado de https://core.ac.uk/download/pdf/42357489.pdf

Bonil, J., Junyent, M., y Pujol, R. M. (2010). Educación para la sostenibilidad desde la perspectiva de la complejidad. Revista Eureka sobre Enseñanza y Divulgación de las Ciencias, 7(número extraordinario), 198-215. Recuperado de https://revistas.uca.es/index.php/eureka/article/view/2644

Bord, R. J. \& O'Connor, R. E. (1997). The gender gap in environmental attitudes: The case of perceived vulnerability to risk. Social Science Quarterly, 78(4), 830-840.

Boubonari, T., Markos, A., \& Kevrekidis, T. (2013). Greek pre-service teachers' knowledge, attitudes, and environmental behavior toward marine pollution. The Journal of Environmental Education, 44(4), $232-251$. https://doi.org/10.1080/00958964.2013.785381

Braun, T., Cottrell, R., \& Dierkes, P. (2018). Fostering changes in attitude, knowledge and behavior: Demographic variation in environmental education effects. Environmental Education Research, 24(6), 899-920. https://doi.org/10.1080/13504622.2017.1343279

Breiting, S. (1997). Hacia un nuevo concepto de educación ambiental. Carpeta informativa del CENEAM, 1-8. Recuperado de https:/www.miteco.gob.es/en/ceneam/articulos-de-opinion/1997soren-breiting_tcm38-163533.pdf_

Brody, M. J. (1996). An assessment of 4th-, 8th-, and 11th-grade students' environmental science knowledge related to Oregon's marine resources. The Journal of Environmental Education, 27(3), 21-27. https://doi.org/10.1080/00958964.1996.9941463

Bybee, R. W. (1991). Planet earth in crisis: How should science educators respond? The American Biology Teacher, 53(3), 146-153. https://doi.org/10.2307/4449248

Cebrián, G. y Junyent, M. (2014). Competencias profesionales en educación para la sostenibilidad: un estudio exploratorio de la visión de futuros maestros. Enseñanza de las Ciencias, 32(1), 29-49. https://doi.org/10.5565/rev/ensciencias.877

Cebrián, G. \& Junyent, M. (2015). Competencies in education for sustainable development: Exploring the student teachers' views. Sustainability, 7(3), 2768-2786. https://doi.org/10.3390/su7032768

Creswell, J. W. (2014). Research design: Qualitative, quantitative, and mixed methods approaches. Los Angeles: Sage publications.

Donahue, A. K. \& Miller, J. M. (2006). Experience, attitudes, and willingness to pay for public safety. The American Review of Public Administration, 36(4), 395-418. https://doi.org/10.1177\%2F0275074005285666

European Commission, EC. (2019). Towards a Sustainable Europe by 2030. Recuperado de https://ec.europa.eu/info/publications/towards-sustainable-europe-2030_en

Ernst, J. \& Theimer, S. (2011). Evaluating the effects of environmental education programming on connectedness to nature. Environmental Education Research, 17(5), 577-598. https://doi.org/10.1080/13504622.2011.565119_

Evans, G. W., Brauchle, G., Haq, A., Stecker, R., Wong, K., \& Shapiro, E. (2007). Young children's environmental attitudes and behaviors. Environment and Behavior, 39(5), 635-658. https://doi.org/10.1177\%2F0013916506294252 
Flores, R. C., Ruiz, M. G., y del Socorro Rayas, J. G. R. (2017). La educación ambiental en la formación docente inicial. Pesquisa em Educação Ambiental, 12(2), 80-92. https://doi.org/10.18675/2177-580x.vol12.n2.p80-92

Forbes, C. T. \& Zint, M. (2010). Elementary teachers' beliefs about, perceived competencies for, and reported use of scientific inquiry to promote student learning about and for the environment. The Journal of Environmental Education, 42(1), 30-42. https://doi.org/10.1080/00958961003674673

García, J. E. (2004). Educación ambiental, constructivismo y complejidad: una propuesta integradora. Sevilla: Díada Editora.

García-Esteban, F. E. y Murga Menoyo, M. Á. (2015). El profesorado de educación infantil ante el desarrollo sostenible: necesidades formativas. Enseñanza \& Teaching, 33(1), 121-142. https://doi.org/10.14201/et2015331121142

GEO-6 (2019). Perspectivas del medio ambiente. Nairobi: Programa de las Naciones Unidas para el Medio Ambiente. Recuperado de https://www.unenvironment.org/es/resources/perspectivas-del-medio-ambiente-mundial-6

Gifford, R. (2014). Environmental psychology matters. Annual Review of Psychology, 65(1), 541-579. https://doi.org/10.1146/annurev-psych-010213-115048

Gifford, R. \& Nilsson, A. (2014). Personal and social factors that influence pro environmental concern and behaviour: A review. International Journal Psychology, 49(3), 141-157. https://doi.org/10.1002/ijop.12034

Gifford, R. \& Sussman, R. (2012). Environmental attitudes. En S. D. Clayton (Ed.), Oxford library of psychology. The Oxford handbook of environmental and conservation psychology (pp. 65-80). Oxford: Oxford University Press. https://doi.org/10.1093/oxfordhb/9780199733026.013.0004

Gisbert, P. (2007). Decrecimiento: camino hacia la sostenibilidad. Recuperado de https://www.ecologistasenaccion.org/13381/el-decrecimiento-camino-hacia-la-sostenibilidad/

Goldsmith, R. E., Feygina, I., \& Jost, J. T. (2013). The gender gap in environmental attitudes: A system justification perspective. En M. Alston \& K. Whittenbury (Eds.), Research, action and policy: Addressing the gendered impacts of climate change (pp. 159-171). Springer, Dordrecht.

González Gaudiano, E. J. (2012). La ambientalización del currículum escolar: breve recuento de una azarosa historia. Profesorado. Revista de Currículum y Formación de Profesorado, 16(2), 15-24. Recuperado de https://recyt.fecyt.es/index.php/profesorado/article/view/43677

Gough, A. (2016). Teacher education for sustainable development: Past, present and future. En W. Leal Filho \& P. Pace (Eds.), Teaching education for sustainable development at university level (pp. 109-122). Berlin: Springer.

Hernández, A. M. , Burgui, M. B., Velázquez, F., y Corrales, J. M. (2018). ¿ Responden los libros de texto a las demandas de la educación ambiental? Un análisis para la educación secundaria. Boletín de la Asociación de Geógrafos Españoles, 77, 80-110.

Heyl, M., Moyano Díaz, E., \& Cifuentes, L. (2013). Environmental attitudes and behaviors of college students: A case study conducted at a Chilean university. Revista Latinoamericana de Psicologia, 45(3), 487-500. https://doi.org/10.14349/rlp.v45i3.1489

Hunter, L. M., Hatch, A., \& Johnson, A. (2004). Cross-national gender variation in environmental behaviors. Social Science Quarterly, 85(3), 677-694. https://doi.org/10.1111/j.0038-4941.2004.00239.x

IBM (2020). IBM SPSS Statistics (Version 20) [Computer software]. Recuperado de https://www.ibm.com/es-es/analytics/spss-statistics-software

Jara Campos, R. (2020). El desempeño de los profesores noveles de ciencias: las competencias profesionales que desarrollan durante los primeros años de ejercicio profesional. Pensamiento Educativo. Revista de Investigación Educacional Latinoamericana, 57(1), 1-18. https://doi.org/10.7764/pel.57.1.2020.2

Jensen, B. B. \& Schnack, K. (2006). The action competence approach in environmental education. Environmental Education Research, 12(3-4), 471-486. https://doi.org/10.1080/13504620600943053

Jones, C., Ramanau, R., Cross, S., \& Healing, G. (2010). Net generation or digital natives: Is there a distinct new generation entering university? Computers \& Education, 54(3), 722-732. https://doi.org/10.1016/j.compedu.2009.09.022

Kerin, R. A., Hartley, S. W., \& Rudelius, W. (2009). Study guide for use with marketing. México, D.F.: McGraw-Hill/Irwin.

Kopnina, H. \& Meijers, F. (2014). Education for sustainable development (ESD) exploring theoretical and practical challenges. International Journal of Sustainability Higher Education, 15(2), 188-207. https://doi.org/10.1108/ijshe-07-2012-0059 
Leal Filho, W., Shiel, C., Paço, A., Mark, M., Veiga Ávila, L., Londero Brandli, L., ... \& Caeiro, S. (2019). Sustainable development goals and sustainability teaching at universities: Falling behind or getting ahead of the pack? Journal of Cleaner Production, 232, 285-294. https://doi.org/10.1016/j.jclepro.2019.05.309

Leal Filho, W., Shiel, C., \& Paço, A. (2016). Implementing and operationalising integrative approaches to sustainability in higher education: The role of project-oriented learning. Journal of Cleaner Production, 133, 126-135. https://doi.org/10.1016/j.jclepro.2016.05.079

Lehnert, M., Fiedor, D., Frajer, J., Hercik, J., \& Jurek, M. (2019). Czech students and mitigation of global warming: Beliefs and willingness to take action. Environmental Education Research, 26(6), 1-26. Recuperado de https://www.tandfonline.com/doi/full/10.1080/13504622.2019.1694140

Leicht, A., Heiss, J., \& Byun, W. J. (2018). Issues and trends in education for sustainable development. Paris: Unesco Publishing.

Lorenzo Rial, M. A., Álvarez Lires, M. M., Arias Correa, A., y Pérez Rodríguez, U. (2019). Aprender a interpretar la acidificación oceánica con recursos on-line y experimentación contextualizada. Enseñanza de las Ciencias, 37(2), 189-208. https://doi.org/10.5565/rev/ensciencias.2564_

Mackey, G. (2012). To know, to decide, to act: The young child's right to participate in action for the environment. Environmental Education Research, 18(4), 473-484. https://doi.org/10.1080/13504622.2011.634494

Marcinkowski, T. \& Reid, A. (2019). Reviews of research on the attitude-behavior relationship and their implications for future environmental education research. Environmental Education Research, 25(4), 459-471. https://www.tandfonline.com/doi/full/10.1080/13504622.2019.1634237

Marquart-Pyatt, S. T. \& Petrzelka, P. (2009). Trust, the democratic process, and involvement in a rural community. Rural Sociology, 73(2), 250-274. https://doi.org/10.1526/003601108784514598

McCright, A. M. \& Xiao, Ch. (2014). Gender and environmental concern: Insights from recent work and for future research. Society \& Natural Resources, 27(10), 1109-1113. https://doi.org/10.1080/08941920.2014.918235

Melero, N. y Solís, C. (2012). Género y medio ambiente. El desafío de educar hacia una dimensión humana del desarrollo sustentable. Revista Internacional de Investigación en Ciencias Sociales, 8(2), 235-250. Recuperado de https://dialnet.unirioja.es/descarga/articulo/4181069.pdf

Milanés, O. A. G., Menezes, P. H. D., y Quellis, L. R. (2019). Educación ambiental transformadora: estudio comparado entre Brasil y Cuba. Revista Pedagógica, 21, 500-523. https://doi.org/10.22196/rp.v22i0.4844

Mogensen, F. \& Schnack, K. (2010). The action competence approach and the 'new' discourses of education for sustainable development, competence and quality criteria. Environmental Education Research, 16(1), 59-74. https://doi.org/10.1080/13504620903504032

Morin, E. (2001). Os sete saberes necessários à educação do futuro. São Paulo: Unesco.

Morin, E. \& Pakman, M. (1994). Introducción al pensamiento complejo. Barcelona: Gedisa.

Müderrisoglu, H. \& Altanlar, A. (2011). Attitudes and behaviors of undergraduate students toward environmental issues. International Journal of Environmental Science \& Technology, 8(1), 159-168.

Murga-Menoyo, M. A. (2015). Competencias para el desarrollo sostenible: las capacidades, actitudes y valores meta de la educación en el marco de la Agenda global pos-2015. Foro de Educación, 13(19), 55-83.

http://dx.doi.org/10.14516/fde.2015.013.019.004

Murga-Menoyo, M. A. y Novo, M. (2017). Sostenibilidad, desarrollo "glocal" y ciudadanía planetaria. Referentes de una pedagogía para el desarrollo sostenible. Teoría de la Educación, 29(1), 55-78. https:/gredos.usal.es/bitstream/handle/10366/134014/Sostenibilidad,_desarrollo_\%ABglocal\%BB_y_ ci.pdf;jsessionid=3DF47EDEAFDC02C5B093AEFDE44B6F59? sequence=1

Novo, M. (2006). El desarrollo sostenible. Su dimensión ambiental y educativa. Madrid: Unesco-Pearson.

Öhman, J. \& Öhman, M. (2012). Participatory approach in practice: An analysis of student discussions about climate change. Environmental Education Research, 19(3), 324-341. https://doi.org/10.1080/13504622.2012.695012

Olsson, D. \& Gericke, N. (2017). The effect of gender on students' sustainability consciousness: A nationwide Swedish study. The Journal of Environmental Education, 48(5), 357-370. https://doi.org/10.1080/00958964.2017.1310083 
Olsson, D., Gericke, N., Boeve-de Pauw, J., Berglund, T., \& Chang, T. (2019). Green schools in Taiwan: Effects on student sustainability consciousness. Global Environmental Change, 54, 184-194. https://doi.org/10.1016/j.gloenvcha.2018.11.011

Olsson, D., Gericke, N., Sass, W., \& Boeve-de Pauw, J. (2020). Self-perceived action competence for sustainability: The theoretical grounding and empirical validation of a novel research instrument. Environmental Education Research, 26(5), 742-760. https://doi.org/10.1080/13504622.2020.1736991

Organización de las Naciones Unidas, ONU. (2015). Transformar nuestro mundo: la Agenda 2030 para el Desarrollo Sostenible. Recuperado de https://sustainabledevelopment.un.org/post2015/transformingourworld

Organización de las Naciones Unidas para la Educación, Unesco. (1999). Declaración sobre la ciencia y el uso del saber cientifico. Recuperado de http://www.unesco.org/science/wcs/esp/declaracion_s.htm

Organización de las Naciones Unidas para la Educación, Unesco. (2016). Informe de Seguimiento de la Educación en el Mundo (Informe GEM): La educación al servicio de los pueblos y el planeta: creación de futuros sostenibles para todos. Recuperado de https://unesdoc.unesco.org/ark:/48223/pf0000248526

Organización de las Naciones Unidas para la Educación, Unesco. (2017). Education for sustainable development goals: Learning objectives. Recuperado de https://unesdoc.unesco.org/ark:/48223/pf0000247444_

Orr, D. (1993). Environmental literacy: Education as if the Earth Mattered. Great Barrington: EF Schumacher Society.

Osorio, S. N. (2002). Aproximaciones a un nuevo paradigma en el pensamiento científico. En M. A. Velilla (Comp.), Manual de iniciación pedagógica al pensamiento complejo (pp. 38-59). Bogotá: Instituto Colombiano para la Evaluación de la Educación, ICFES.

Pavalache-Ilie, M. \& Cazan, A. M. (2018). Personality correlates of pro-environmental attitudes. International Journal of Environmental Health Research, 28(1), 71-78. https://doi.org/10.1080/09603123.2018.1429576

Pe'er, S., Goldman, D., \& Yavetz, B. (2007). Environmental literacy in teacher training: Attitudes, knowledge, and environmental behavior of beginning Students. The Journal of Environmental Education, 39, 45-59. https://doi.org/10.3200/JOEE.39.1.45-59

Pérez-Rodríguez, U., Varela-Losada, M., Álvarez Lires, F. J., \& Vega-Marcote, P. (2017). Attitudes of preservice teachers: Design and validation of an attitude scale toward environmental education. Journal of Cleaner Production, 164, 634-641. http://dx.doi.org/10.1016/j.jclepro.2017.06.245

Pérez-Rodríguez, U., Varela-Losada, M., Lorenzo-Rial, M.A., y Vega-Marcote, P. (2017). Tendencias actitudinales del profesorado en formación hacia una educación ambiental transformadora. Revista de Psicodidáctica, 22(1), 60-68. https://doi.org/10.1016/s1136-1034(17)30045-x

Phillips, D. C. \& Burbules, N.C. (2000). Post positivism and educational research. Lanham: Rowman \& Littlefield Publishers.

Rieckmann, M. (2012). Future-oriented higher education: Which key competencies should be fostered through university teaching and learning? Futures, 44(2), 127-135. https://doi.org/10.1016/j.futures.2011.09.005

Rockström, J. \& Klum, M. (2015). Big world, small planet: Abundance within planetary boundaries. New Haven: Yale University Press.

Rockström, J., Steffen, W., Noone, K., Persson, Å., Chapin III, F. S., Lambin, E., ... \& Foley, J. (2009). Planetary boundaries: Exploring the safe operating space for humanity. Ecology and Society, 14(2), 1-32. https://doi.org/10.5751/es-03180-140232

Rodríguez, F. (2011). Educación ambiental para la acción ciudadana: concepciones del profesorado en formación sobre la problemática de la energía (Tesis doctoral). Universidad de Sevilla, Sevilla.

Rodríguez, F. y García, J. E. (2009). El activismo que no cesa. Obstáculos para incorporar la metodología didáctica basada en la investigación del alumno a la práctica de la educación ambiental. Revista Investigación en la Escuela, 67, 23-36. Recuperado de https://idus.us.es/handle/11441/60787

Saheb, D. \& Rodrigues, D. G. (2017). A contribuição da complexidade de Morin para as pesquisas em educação ambiental. REMEA-Revista Eletrônica do Mestrado em Educação Ambiental, Edição Especial XVI Encontro Paranaense de Educação Ambiental, 191-207.

Schindel Dimick, A. (2015). Supporting youth to develop environmental citizenship within/against a neoliberal context. Environmental Education Research, 21(3), 390-402. https://doi.org/10.1080/13504622.2014.994164 
Scott, W. (2009). Environmental education research: 30 years on from Tbilisi. Environmental Education Research, 15(2), 155-164. https://doi.org/10.1080/13504620902814804

Skamp, K. (2009). Understanding teachers' 'levels of use' of learnscapes. Environmental Education Research, 15(1), 93-110. https://doi.org/10.1080/13504620802629864

Solbes, J. y Torres, N. (2012). Análisis de las competencias de pensamiento crítico desde el aborde de las cuestiones sociocientíficas: un estudio en el ámbito universitario. Didáctica de las Ciencias Experimentales y Sociales, 26, 247-269. https://doi.org/10.7203/dces.26.1928

Steffen, W., Broadgate, W., Deutsch, L., Gaffney, O., \& Ludwig, C. (2015). The trajectory of the Anthropocene: The great acceleration. The Anthropocene Review, 2(1), 81-98. https://doi.org/10.1177/2053019614564785

Steffen, W., Richardson, K., Rockström, J., Cornell, S. E., Fetzer, I., Bennett, E. M., ... \& Folke, C. (2015). Planetary boundaries: Guiding human development on a changing planet. Science, 347(6223), 1259855-1259855. https://doi.org/10.1126/science.1259855

Stevenson, K. T., Peterson, M. N., \& Bondell, H. D. (2019). The influence of personal beliefs, friends, and family in building climate change concern among adolescents. Environmental Education Research, 25(6), 832-845. https://doi.org/10.1080/13504622.2016.1177712

Stiglitz, J. E. (2015) La gran brecha: qué hacer con las sociedades desiguales. Madrid: Taurus.

Tehrani, S. M., Karbassi, A. R., Monavari, S. M., \& Mirbagheri, S. A., (2010). Role of e-shopping management strategy in urban environment. International Journal Environmental Research, 4(4), 681-690. https://dx.doi.org/10.22059/ijer.2010.254

Thapa, B. (1999). Environmentalism: A study of undergraduate students. En G. Kyle (Comp./Ed.), Proceedings of the 1999 Northeastern recreation research symposium. New York: Bolton Landing. https://doi.org/10.2737/NE-GTR-269

Tilbury, D. (1995). Environmental education for sustainability: Defining the new focus of environmental education in the 1990s. Environmental Education Research, 1(2), 195-212. https://doi.org/10.1080/1350462950010206

Tilbury, D. (2011). Assessing ESD Experiences during the DESD: An expert review on processes and learning for ESD. Recuperado de http://unesdoc.unesco.org/images/0019/001914/191442e.pdf

Torres, N. y Solbes, J., (2016) Contribuciones de una intervención didáctica usando cuestiones sociocientíficas para desarrollar el pensamiento crítico. Enseñanza de las Ciencias, 34(2), 43-65. Recuperado de https://www.raco.cat/index.php/Ensenanza/article/view/v34-n2-torres-solbes/399258

Treagust, D. F., Won, M., \& Duit, R. (2014). Paradigms in science education research. En N. G. Lederman \& S. K. Abell (Eds.), Handbook of research on science education, volume II (pp. 17-31). London: Routledge.

Tuncer Teksoz, G., Boone, J. W., Yilmaz Tuzun, O., \& Oztekin, C. (2014). An evaluation of the environmental literacy of preservice teachers in Turkey through Rasch analysis. Environmental Education Research, 20(2), 202-227. https://doi.org/10.1080/13504622.2013.768604

Ull, M. A., Martínez-Agut, M. P., Piñero, A., \& Aznar-Minguet, P. (2014). Perceptions and attitudes of students of teacher-training towards environment and sustainability. Procedia Social Behavior Science, 131, 453-457. https://doi.org/10.1016/j.sbspro.2014.04.147

Ull, M. A., Pińero, A., Martínez-Agut, M. P., y Aznar, P. A. (2014). Preconcepciones y actitudes del profesorado de magisterio ante la incorporación en su docencia de competencias para la sostenibilidad. Enseñanza de las Ciencias: Revista de Investigación y Experiencias Didácticas, 32(2), 91-112. Recuperado de https://www.raco.cat/index.php/Ensenanza/article/view/v32-n2-ull-pinero-martinez-agut-aznar/375680

Universidade de Vigo. (2018). Guia de boas prácticas en Investigación da Universidade de Vigo. Recuperado de https://www.uvigo.gal/sites/uvigo.gal/files/contents/paragraph-file/2018-04/Guia\%20boas\%20practicas\%20 investigaci\%C3\%B3n.pdf

Van Petegem, P., Blieck, A., Imbrecht, I., \& Van Hout, T. (2005). Implementing environmental education in pre-service teacher training. Environmental Education Research, 11(2), 161-171. https://doi.org/10.1080/1350462042000338333

Vega-Marcote, P. \& Álvarez, P. (2012). Training of teachers in Spain towards sustainability. Implementation and analysis of ecomethodology. European Journal of Teacher Education, 35(4), 494-510. https://doi.org/10.1080/02619768.2011.643400

Vilches, A. y Gil, D. (2009). Una situación de emergencia planetaria, a la que debemos y "podemos" hacer frente. Revista de Educación, 1, 101-122. Recuperado de https://www.oei.es/historico/decada/re2009_05.pdf 
Vilches, A. y Gil, D. (2015). Ciencia de la sostenibilidad: ¿¿Una nueva disciplina o un nuevo enfoque para todas las disciplinas? Revista Iberoamericana de Educación, 69(1), 39-60. https://doi.org/10.35362/rie691152

Wals, A. E. (2014). Sustainability in higher education in the context of the UN DESD: A review of learning and institutionalization process. Journal of Cleaner Production, 62, 8-15. http://dx.doi.org/10.1016/j.jclepro.2013.06.007

Wiek, A., Withycombe, L., \& Redman, C. L. (2011). Key competencies in sustainability: A reference framework for academic program development. Sustainability Science, 6(2), 203-218. https://doi.org/10.1007/s11625-011-0132-6

Wiek, A., Xiong, A., Brundiers, K., \& Leeiw Van Deer, S. (2014). Integrating problem-and project- based learning into sustainability programs. A case study on then School of Sustainability at Arizona State University. International Journal of Sustainability in Higher Education, 15(4), 413-449. https://doi.org/10.1108/ijshe-02-2013-0013

Winter, D. D. \& Koger, S. M. (2008). The psychology of environmental problems: Psychology for sustainability. New York: Psychology press.

Worldwatch Institute (2015). State of the World 2015: Confronting hidden threats to sustainability. Island Press.

Yavetz, B., Goldman, D., \& Pe'er, S. (2009). Environmental literacy of pre-service teachers in Israel: A comparison between students at the onset and end of their studies. Environmental Education Research, 15(4), 393-415. https://doi.org/10.1080/13504620902928422

Yurt, Ö., Cevher-Kalburan, N., \& Kandır, A. (2010). WCES-2010 Investigation of the environmental attitudes of the early childhood teacher candidates. Procedia-Social and Behavioral Sciences, 2(2), 4977-4984. https://doi.org/10.1016/j.sbspro.2010.03.806

Yus Ramos, R. (1993). Entre la cantidad y la calidad. Cuadernos de Pedagogía, 220, 64-77. 


\section{Apéndice}

Escala de actitudes hacia la educación ambiental (Attitudes Scale toward Environmental Education, ASEE) (Pérez-Rodríguez, Varela-Losada, Álvarez-Lires \& Vega-Marcote, 2017).

Datos básicos

\begin{tabular}{ll}
\hline Edad: $\quad \square$ Menos de 18 años $\square$ 18-22 años $\quad \square$ 22-30 años $\square$ Más de 30 años \\
\hline Sexo: $\quad \square$ Hombre $\square$ Mujer \\
\hline Titulación: $\square$ Grado en Educación Primaria $\square$ Grado en Educación Infantil \\
\hline Curso (marcar solo el mayor): $\quad \square 1^{\circ} \quad \square 2^{\circ} \quad \square 3^{\circ} \quad \square 4^{\circ}$ \\
\hline Bachillerato cursado: & $\square$ Artes $\square$ Ciencias/Tecnología $\square$ Humanidades y Ciencias Sociales \\
\hline Centro de procedencia: & $\square$ Público $\square$ Concertado laico $\square$ Concertado religioso \\
& $\square$ Privado laico $\square$ Privado religioso \\
\hline Nivel de estudios de la madre: & $\square$ Sin estudios $\square$ Básicos $\square$ Secundarios \\
& $\square$ Superiores \\
Nivel de estudios del padre: & $\square$ Sin estudios $\square$ Básicos $\square$ Secundarios \\
& $\square$ Superiores
\end{tabular}

CUESTIONARIO. Lee con atención las siguientes afirmaciones y señala tu grado de acuerdo o desacuerdo

i1 Ante los problemas ambientales de nuestro tiempo es prioritario integrar la educación ambiental en la escuela

i2* Considero que no es prioritario que la educación ambiental trate el modelo socioeconómico actual basado en el consumo

i3 La educación ambiental debería trabajar especialmente el desarrollo de habilidades como el pensamiento crítico, la toma de decisiones reflexivas y la participación

i4* Considero que analizar problemas ambientales y buscar soluciones es demasiado complejo para el alumnado de primaria

i5* El alumnado pierde demasiado tiempo en buscar y analizar la información, es mucho más provechoso facilitarles la información ya seleccionada y analizada

i6 Para que la educación ambiental sea lo más efectiva posible debería existir un compromiso de toda la comunidad educativa

i7* No me parece que el comportamiento del profesorado sea un factor muy importante en el aprendizaje de valores ambientales

i8 Creo que incluir la educación ambiental en la escuela puede contribuir a cambiar el comportamiento ambiental de toda la comunidad

i9 Me parece importante que todo el profesorado reciba formación ambiental

i10* Creo que individualmente no tengo poder para solucionar los problemas ambientales

i11* El mejor indicador de la prosperidad de un país es su crecimiento económico

i12* Creo que el factor que más determina el bienestar de las personas es su renta

i13* Prefiero no saber cómo se han producido todos los bienes que consumo

i14* Prefiero un producto más barato aunque piense que se ha producido de forma irresponsable

i15* La gravedad del cambio climático ha sido exagerada

i16* Creo que el efecto del cambio climático sobre mi vida no es importante.

i17* La contaminación debida a la producción de energía es un mal menor, frente a los beneficios que reporta

i18* Me parece que utilizar el coche en desplazamientos privados no supone un gran aumento de los gases que producen el cambio i18* climático

Nota: Los asteriscos indican que las respuestas al ítem se recodifican invirtiendo su orden.

Fuente: Elaboración propia 